\title{
Nurses are the Backbone of a Healthcare System
}

\author{
Shaheena Salman Alwani ${ }^{*}$ \\ ${ }^{1}$ Section Head-Clinical Nursing, Liaquat National Hospital and Medical College, Karachi, Pakistan
}

A Nurse plays a pivotal role in any health care system. Without which effective coordination and collaboration seem dreadful. They would bridge the gap between health care practitioners and patients. Thus, they work as the backbone for any Health care system [1].

The role of a nurse, over time, has become more diverse. Based on the current pandemic of the COVID-19 outbreak, nurses are the ones assisting the doctors and reaching out to patients with significant information to disseminate, handling that information, administering care, uplifting the spirits, and preventing the disease [1]. Not only are nurses vital to patient care, but they are also members of the administration and management team [2] and in this year of 2020, global contributions and efforts of the Nurses are evidently seen, keeping their health on a verge of counteracting the burden of the disease, without thinking for their own lives, nurses have come upfront to save millions of people around battling with the catastrophic disease of the era.

For improving the hospital's quality, the nurse's contribution is markedly prominent. Any hospital's image is sorted through the Nursing force's indulgence in satisfying care levels to patients. Nurses not only provide bedside care but eventually have accrued positions in the hospital management hierarchy. They not only assist doctors in surgeries but follow through with the care till the patient's home. They train and oversee other nurses and educate patients and family members regarding continuing and post-hospital care during discharge [2]. The diverse role of a nurse can be articulated from performing a physical examination, obtaining health histories, counseling the patients, advocating for the rights of patients and colleagues, interpreting patient information and making critical decisions about needed actions, conducting research in support of improved practice and patient outcomes [3]. To add on, during COVID-19 offering self to have home health visits and tele-health encounters is yet a new dimension and an unexplored Nursing paradigm in many regions of Pakistan.

Any organization that fully encompasses Nursing contributions, would boom and flourish extensively.

*Corresponding Author: Shaheena Salman Alwani, Section Head-Clinical Nursing, Liaquat National Hospital and Medical College, Karachi, Pakistan; Email:Shaheena.salman@Inh.edu.pk

Received: November 30, 2020; Revised:December 07, 2020; Accepted: December 11, 2020 DOI: https://doi.org/10.37184/lnjpc.2707-3521.2.10
Work motivation to Nurses is critical for accomplishing the organization's goals and benefiting patients [4]. The autonomous power for the Nursing fraternity is the need of the time especially in underdeveloped countries along with monetary and contingent rewards/ appreciations for this profession.

\section{REFERENCES}

1. Tampa Bay Newswire. Why nurses are the backbone of a healthcare system. Available at: https://www.tampabaynewswire.com/2020/06/12/ why-nurses-are-the-backbone-of-a-healthcare-system-87002.

2. ATHENA Career Academy. 3 factors that make nurses the backbone of hospital. Available at: https://info.athenacareers. edu/blog/3-factors-that-make-nurses-the-backbone-of-hospitals.

3. Rosa B. What nurses really contribute to health care: part 1 of an education do-over.

4. Baljoon RA, Banjar HE, Banakhar MA. Nurses' work motivation and the factors affecting it: a scoping review. Int J Nursing Clin Pract 2018; 5: 1-10. 